\title{
Forsythiae Fructus and Its Active Component, Arctigenin, Provide Neuroprotection by Inhibiting Neuroinflammation
}

\author{
Ji-Ho Park ${ }^{1, a}$, Ye-Ji Hong ${ }^{1, a}$, Eunjung Moon ${ }^{1}$, Seul-A Kim ${ }^{1}$ and Sun Yeou Kim ${ }^{1,2, *}$ \\ ${ }^{1}$ Graduate School of East-West Medical Science, ${ }^{2}$ East-West Integrated Medical Science Rearch Center, \\ Kyung Hee University Global Campus, Yongin 446-701, Republic of Korea
}

\begin{abstract}
In this study, we found that Forsythiae fructus (FF) and one of its main compounds, arctigenin, significantly inhibited nitric oxide production in lipopolysaccharide (LPS)-stimulated BV-2 microglial cells. Arctigenin also suppressed the expression of inducible nitric oxide synthase and cyclooxygenase-2, and inhibited the activation of extracellular signal-regulated kinase, c-Jun $\mathrm{N}$-terminal kinase and p38. Moreover, it also reduced levels of proinflammatory cytokines, interleukin $1 \beta$, tumor necrosis factor $\alpha$ and prostaglandin E2, and inhibited neuronal death in LPS-treated organotypic hippocampal cultures. Therefore, we suggest that arctigenin may confer a neuroprotective effect via the inhibition of neuroinflammation.
\end{abstract}

Key Words: Forsythiae fructus, Arctigenin, Microglia, Neuroprotection, Neuroinflammation

\section{INTRODUCTION}

Microglia are the resident immune cells of the brain. Under normal conditions, they play important roles in the regulation of homeostasis and defense against injury (Perry and Gordon, 1988). However, in pathological conditions, microglia are over-activated and produce reactive proinflammatory mediators, including nitric oxide (NO), interleukin $1 \beta$ (IL-1 $\beta$ ), tumor necrosis factor $\alpha($ TNF- $\alpha)$ and prostaglandin $\mathrm{E}_{2}\left(\mathrm{PGE}_{2}\right)$ (Minghetti and Levi, 1998; Hanisch, 2002). These also play a deleterious role in neuronal death and neurodegenerative diseases, such as Alzheimer's disease and Parkinson's disease (Boje and Arora, 1992; Banati et al., 1993; Gehrmann et al., 1995). Therefore, much attention has been focused on materials that could effectively regulate the activation of microglia. Previously, we demonstrated that apigenin (Ha et al., 2008), falcarindiol (Kim et al., 2003a) and 15,16-dihydrotanshinone (Lee et al., 2006) can act as inhibitors of LPS-induced overstimulation in microglia.

In Korea, Forsythiae fructus (FF; Forsythia koreana Nakai, Oleaceae) is a well-known traditional herbal medicine that has been used for the treatment of inflammation, as well as a diuretic and an antibacterial. Some phytochemical investigations of FF have previously been conducted. Several constituent substances of FF were reported, including lignans, phenylethanoid glycosides and flavonols (Guo et al., 2007; Piao et al., 2008).
Arctigenin is a phenylpropanoid dibenzylbutyrolactone lignan found in FF with anti-virus and anti-tumor pharmacological activities (Yang et al., 2005; Awale et al., 2006). Antiinflammatory activities of FF and arctigenin have previously been reported, and FF inhibits allergic inflammatory reactions through the downregulation of mast cell activation (Kim et al., 2003b; Choi et al., 2007). Moreover, it inhibits NO production and inducible nitric oxide synthase (iNOS) expression in Raw264.7 cells (Kim et al., 2000). Arctigenin also has significant anti-inflammatory and analgesic activities in animal models of acute inflammation (Kang et al., 2008). It also inhibits B- and T-cell mediated allergic inflammation, as well as proinflammatory enzymes, such as cyclooxygenase-1 (COX-1) and COX-2, 5-lipoxygenase, phospholipase A2 and phosphodiesterase (Lee and Kim, 2010).

The purpose of this study was to investigate the inhibitory activities of FF and arctigenin on neuroinflammation and to determine the neuroprotective effects of arctigenin.

\section{MATERIALS AND METHODS}

\section{Materials}

FF was obtained from Omniherb (Daegu, Korea). Dulbecco's modified Eagle's medium (DMEM), minimum essential medium (MEM), fetal bovine serum (FBS), horse serum (HS), penicillin-streptomycin (PS), Hank's buffered salt solution

Received Jun 3, 2011 Revised Aug 9, 2011 Accepted Aug 9, 2011

\footnotetext{
*Corresponding Author

E-mail: sunnykim@khu.ac.kr

Tel: +82-31-201-2177, Fax: +82-31-205-8962

a Ji-Ho Park and Ye-Ji Hong contributed equally to this study.
} 
(HBSS) and 4-(2-hydroxyethyl)-1-piperazineethanesulfonic acid (HEPES) were purchased from Invitrogen (Carlsbad, CA, USA). Lipopolysaccharide (LPS) and arctigenin were obtained from Sigma Chemical Company (St. Louis, MO, USA).

\section{Sample preparation and high performance liquid chro- matographic analysis}

FF (1 kg) were extracted with $85 \%$ ethanol in water using an ultrasonic apparatus and then concentrated under vacuum. In total, $110 \mathrm{~g}$ of ethanol extracts (FF-T) were isolated. FF-T was suspended in water and partitioned with ethylacetate. The aqueous layer was partitioned with $n$-butanol. The total yields of ethylacetate-soluble (FF-E) and $n$-butanol-soluble fraction (FF-B) from FF were $6.7 \%(67.3 \mathrm{~g})$ and $1.1 \%(11.0 \mathrm{~g})$, respectively. The water-soluble fraction (FF-W) was $18.7 \mathrm{~g}$. Arctigenin (Sigma-Aldrich Co. Ltd., St. Louis MO, USA) in FF-E was quantified by high performance liquid chromatographic analysis (HPLC; Agilent Technologies Inc., Santa Clara, CA, USA) under the following conditions: column, J'sphere ODSH80 column $(250 \times 4.6$ mm, $4 \mu \mathrm{m}, \mathrm{YMC}$ Co. Ltd., Ishikawa, Japan); detector wavelength, $280 \mathrm{~nm}$; flow rate, $1.0 \mathrm{ml} / \mathrm{min}$; and retention time, 17.94 minutes. The mobile phase consisted of (A) acetonitrile and $(B)$ water, and each contained $0.02 \%$ of trifluoroacetic acid. The following gradient elution was used: 0 to 10 minutes, $90 \%$ B; 20 minutes, $70 \%$ B; and 30 minutes, $0 \%$ $B$. The arctigenin content in FF-E was determined using the linear regression equation from the calibration graph, and the arctigenin content in FF-E was $36.80( \pm 0.07) \mathrm{mg} / \mathrm{g}$ (Fig. 1C).

\section{Nitric oxide (NO) and cell viability assay}

The BV-2 mouse microglial cell line was maintained in DMEM supplemented with $5 \%$ FBS and $1 \%$ PS. To measure NO production, cells were plated in a 96-well plate $\left(3 \times 10^{4}\right.$ cells/well). After $24 \mathrm{~h}$, cells were pretreated with samples for 30 minutes and then stimulated with $100 \mathrm{ng} / \mathrm{ml}$ of LPS for another $24 \mathrm{~h}$. LPS triggers an innate immune responses in microglia through Toll-like receptor 4 (TLR4), which participates in pathogen recognition (Miyake, 2004). Therefore, it has been widely used as an activator of microglia.

Nitrite, a soluble oxidation product of NO, was measured in the culture media using Griess reagents ( $1 \%$ sulfanilamide, $0.1 \% \mathrm{~N}$-1-napthylethylenediamine dihydrochloride in $5 \%$ phosphoric acid). Cell viability was assessed by a 3-[4, 5-dimethylthiazol-2-yl]-2, 5-diphenyl-tetrazolium bromide (MTT) assay.

\section{Western blot analysis}

We performed Western blot analysis to assess the effects of arctigenin on the protein expression of iNOS and COX-2 and the phosphorylation of ERK, JNK and p38. BV-2 cells were seeded in a six-well plate $\left(2 \times 10^{6}\right.$ cells/well). After $24 \mathrm{~h}$, cells were pretreated with arctigenin for 30 minutes and then exposed to LPS (100 ng/ml) for $6 \mathrm{~h}$ (iNOS and COX-2) and 30 minutes (ERK, JNK and p38). Protein samples from cell extracts were separated by $8 \%$ SDS-PAGE and transferred to a nitrocellulose membrane. The membrane was blocked with $5 \%$ skim milk and then incubated with primary antibodies (Cell Signaling, Danver, MA, USA) and horseradish peroxidaseconjugated secondary antibodies (Santa Cruz Biotechnology, Santa Cruz, CA, USA). The blots were developed using ECL Western Blotting Detection Reagents (Amersham Bioscience, Piscataway, NJ, USA).

\section{Enzyme-linked immunosorbent assay}

For determining the effects of arctigenin on proinflammatory cytokines, BV-2 cells were pretreated with arctigenin for 30 minutes, and then inflammation of cells was induced with LPS $(100 \mathrm{ng} / \mathrm{ml})$. After $24 \mathrm{~h}$, the supernatant from the culture medium was used to determine the levels of IL-1 $\beta$, TNF- $\alpha$ and $\mathrm{PGE}_{2}$ as measured by enzyme immunoassay kits (R\&D Systems, Minneapolis, MN, USA) according to the manufacturer's protocol.

\section{Organotypic hippocampal culture}

The experimental procedure was approved by the Animal Research Committee of the Kyung Hee University (2008-006). All efforts were made to minimize the number of animals used in this study and their suffering. We isolated the hippocampus of the brains of one-day-old Sprague-Dawley rats, after which the dorsal halves were sectioned into $350 \mu \mathrm{m}$-thick slices using a tissue chopper (Mickle Laboratory Engineering Co., Surrey, UK). The slices were placed on a membrane insert $(0.4$ $\mu \mathrm{m}$, Millicell-CM, Milliopore, Bedford, MA, U.S.A) that was set into six-well plates filled with MEM medium containing $1 \% \mathrm{PS}$, $25 \% \mathrm{HS}, 25 \%$ HBSS, $6 \mathrm{~g} / \mathrm{L}$ D-glucose, $1 \mathrm{mM}$ L-glutamine and $20 \mathrm{mM}$ HEPES. After two weeks, the tissues were pretreated with arctigenin for 30 minutes and then exposed to $10 \mu \mathrm{g} / \mathrm{ml}$ of LPS for $72 \mathrm{~h}$. We stained the tissues with propidium iodide (PI; Piao et al., 2008) in order to assess neuronal cell death. PI fluorescent imaging analysis was performed using a fluorescence microscope (Carl Zeiss, Goettingen, Germany) at $514 \mathrm{~nm}$.

\section{Statistical analysis}

All data are expressed as the mean $( \pm$ SE) of at least three independent experiments. Statistical comparisons between the different treatments were performed using one-way ANO$V A$ with Student's $t$-test. Results with $p$-values $<0.05$ were considered statistically significant.

\section{RESULTS}

\section{FF and arctigenin inhibited NO production in BV-2 cells}

We measured NO production after treatment with FF extract (FF-T), its subfractions (FF-E, FF-B and FF-W) and arctigenin in LPS-activated BV-2 cells. NO production was inhibited in cells treated with FF-T compared to cells treated with LPS alone without cell toxicity. FF-B had no effect on NO production, and FF-W did not influence NO levels in the culture medium. However, FF-W at $20 \mu \mathrm{g} / \mathrm{ml}$ was cytotoxic in BV-2 cells. Among the subfractions of FF extract, only FF-E significantly reduced the levels of secreted $\mathrm{NO}$ without cell toxicity (Fig. 1A). Moreover, concentrations of arctigenin ranging from 1 to $5 \mu \mathrm{M}$ also inhibited NO production in a dose-dependent manner (Fig. 1B). To determine whether a large quantity of arctigenin may be contained in FF-E, the most active subfraction of FF-T, we measured the arctigenin content in FF-E by HPLC analysis. Arctigenin was detected as one of the most abundant compounds in FF-E (Fig. 1C).

Arctigenin suppressed the expression of iNOS and COX-2, the secretion of IL-1 $\beta$, TNF- $\alpha$ and PGE ${ }_{2}$ and the activation of MAPKs in BV-2 cells

Two important inflammatory mediators, $\mathrm{NO}$ and $\mathrm{PGE}_{2}$, are 

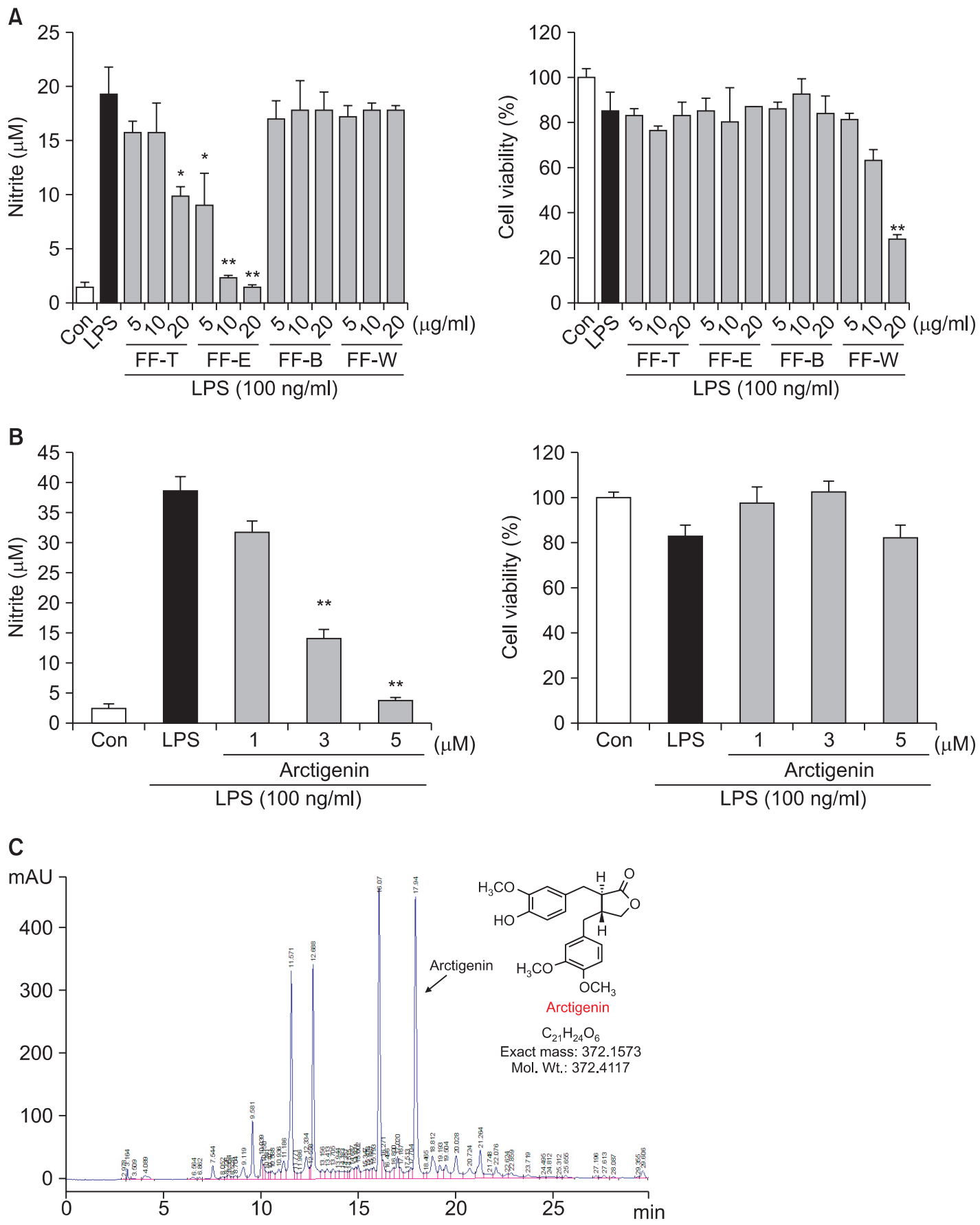

Fig. 1. The effects of FF and arctigenin on NO production in BV-2 cells. The effects of FF (A) and arctigenin (B) on NO production and cell viability. BV-2 cells were pretreated with FF extracts for 30 minutes and then stimulated with LPS (100 $\mathrm{ng} / \mathrm{ml})$ for $24 \mathrm{~h}$. Nitrite was measured using a Griess reaction in culture medium. Cell viability was evaluated using an MTT assay. FF-T, total extract of FF; FF-E, ethylacetate-soluble fraction of FF-T: FF-B, $n$-butanol-soluble fraction of FF-T: and FF-W, water-soluble fraction of FF-T. All data are presented as the mean ( \pm SE) of three independent experiments. ${ }^{*} p<0.05$ and ${ }^{* *} p<0.001$ indicate significant differences when compared to treatment with LPS alone. (C) The HPLC spectrum of arctigenin in FF-E.

produced by iNOS and COX-2, primarily in response to proinflammatory stimulation. We performed Western blot analysis to determine whether arctigenin had an effect on the expression levels of iNOS and COX-2. The pretreatment of cells with arctigenin led to a significant decrease in iNOS protein levels at $3 \mu \mathrm{M}$ in LPS-activated BV-2 cells. Similarly, pretreatment with arctigenin inhibited the expression of COX-2 protein at 5 $\mu \mathrm{M}$ (Fig. 2A).

Mitogen-activated protein kinases (MAPKs) signaling regulates the expression of many genes encoding inflammatory mediators (Kaminska, 2005). Thus, we intended to determine the effect of arctigenin on the phosphorylation of three 
A

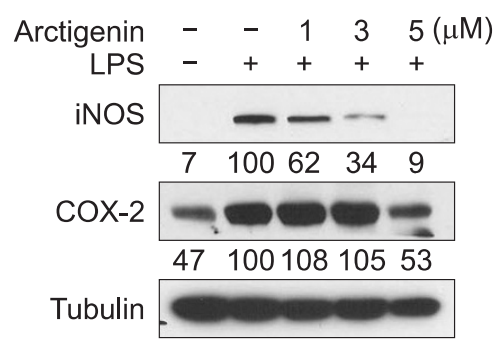

B

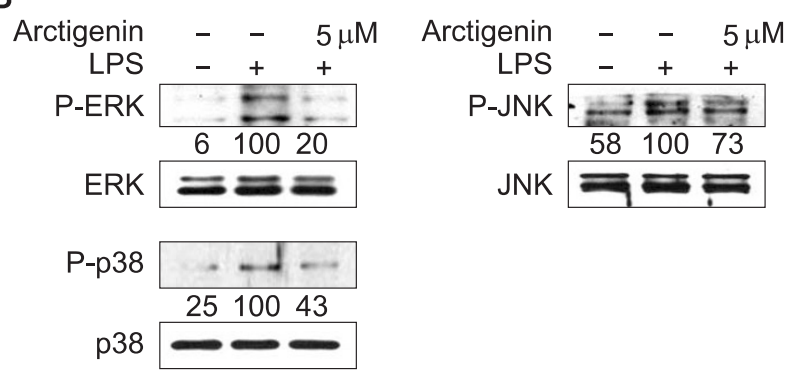

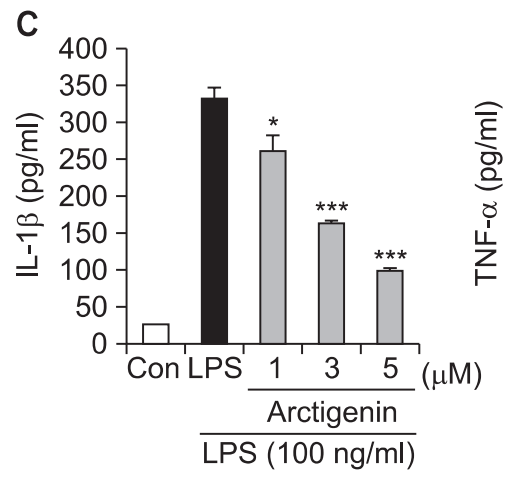
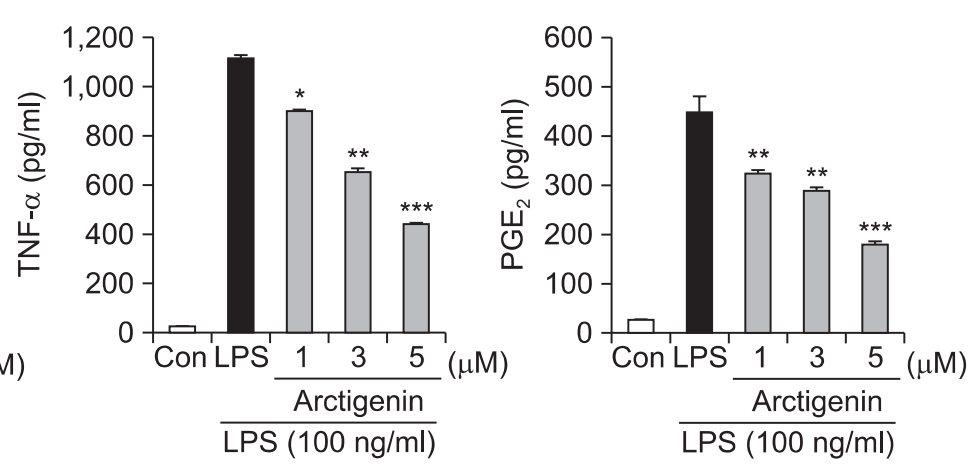

Fig. 2. The effects of arctigenin on the expression of iNOS and COX-2, the activation of MAPKs, and secretion levels of IL-1 $\beta$, TNF- $\alpha$ and $\mathrm{PGE}_{2}$ in BV2 cells. BV-2 cells were pretreated with samples for 30 minutes and then stimulated with LPS (100 $\left.\mathrm{ng} / \mathrm{ml}\right)$ for $6 \mathrm{~h}$ (iNOS and COX-2) and 30 minutes (MAPKs). The effects of arctigenin on LPS-induced iNOS and COX-2 expression (A) and MAPKs activation (B) were determined using Western blot analysis. Densitometry analysis of bands was performed as described in the methods section. The levels of secreted IL-1 $\beta$, TNF- $\alpha$ and $\mathrm{PGE}_{2}(\mathrm{C})$ were assessed using an enzyme immunoassay kit after treatment of BV-2 cells with LPS (100 $\mathrm{ng} / \mathrm{ml})$ for $24 \mathrm{~h}(\mathrm{IL}-1 \beta$ and TNF- $\alpha)$ and $6 \mathrm{~h}\left(\mathrm{PGE}_{2}\right)$ in the presence or absence of arctigenin. All data are presented as the mean $( \pm S E)$ of three independent experiments. ${ }^{*} p<0.05,{ }^{* *} p<0.01$ and ${ }^{* * *} p<0.001$ indicate significant differences as compared to treatment with LPS alone.

MAPKs: ERK, JNK and p38. Treatment with LPS stimulated the phosphorylation of p38, ERK and JNK. However, LPSinduced MAPKs activation was inhibited by $5 \mu \mathrm{M}$ of arctigenin in BV-2 cells (Fig. 2B).

We also measured the levels of proinflammatory cytokines, such as IL-1 $\beta$, TNF- $\alpha$ and $\mathrm{PGE}_{2}$, after treatment with LPS alone and LPS plus arctigenin. The stimulation of microglia by LPS led to an increase in IL-1 $\beta$, TNF- $\alpha$ and $\mathrm{PGE}_{2}$ production. The levels of these cytokines were significantly decreased in a dose-dependent manner when pretreated with arctigenin (Fig. 2C).

\section{Arctigenin reduced neuronal cell death in organotypic hippocampal cultures}

We prepared rat hippocampal slice cultures, treated them with arctigenin after induction of neuronal damage by LPS, and measured the levels of cell death by detecting the level of PI uptake. Excessive PI uptake was only evident in the LPStreated cultures. In contrast, arctigenin inhibited uptake at 0.1 and $0.5 \mu \mathrm{M}$ in a dose-dependent manner (Fig. 3).

\section{DISCUSSION}

Neuroinflammation is associated with neuronal cell death and various neurodegenerative diseases. Our study showed that arctigenin inhibited neuroinflammation induced by overactivated microglia by suppressing NO production, expression of iNOS and COX-2, activation of MAPKs and secretion of proinflammatory cytokines. Moreover, arctigenin also significantly reduced neuronal cell death. Therefore, arctigenin may act as a neuroprotector by inhibiting neuroinflammation, and could be a candidate for treating neurodegenerative diseases.

In this study, we investigated the inhibitory activities of FF and arctigenin on neuroinflammation using the murine microglial cell line BV-2. A previous study showed that arctigenin reduced neuronal death and secondary inflammation and oxidative stress resulting from microglial activation in mice with Japanese encephalitis (Swarup et al., 2008). These results cannot be directly compared to those of our study; however, the effects of arctigenin on inflammation in LPS-induced neuroinflammation animal models are analogous.

In order to determine the effect of FF and arctigenin on neuronal cell death, we used LPS in organotypic hippocampal culture. LPS is a potent activator of microglia and an inducer of neuroinflammation in many in vitro and in vivo experimental models. The organotypic hippocampal slice culture preserves the neuronal environment with the interaction between glial and neuronal cells. Thus, it can be used as a suitable experimental model that reflects in vivo conditions (Frotscher et al., 1990; Frotscher et al., 1995). Therefore, we think the organotypic hippocampal culture treated with LPS is adequate for estimating the anti-neuroinflammatory activity of arctigenin in vivo. In our study, lower concentrations of arctigenin reduced neuronal cell death in primary organotypic hippocampal cultures than were necessary in BV-2 cells. This could be be- 
A

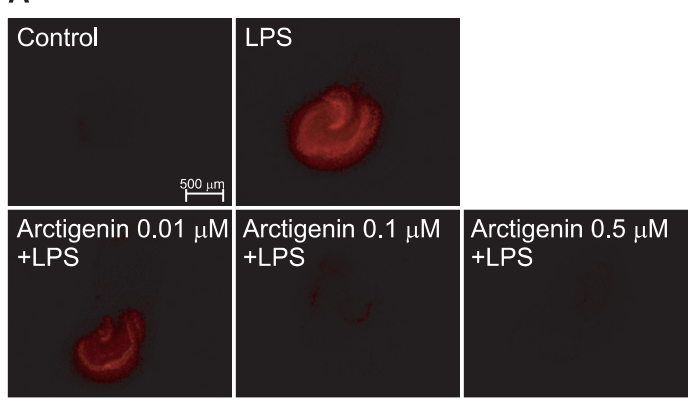

B

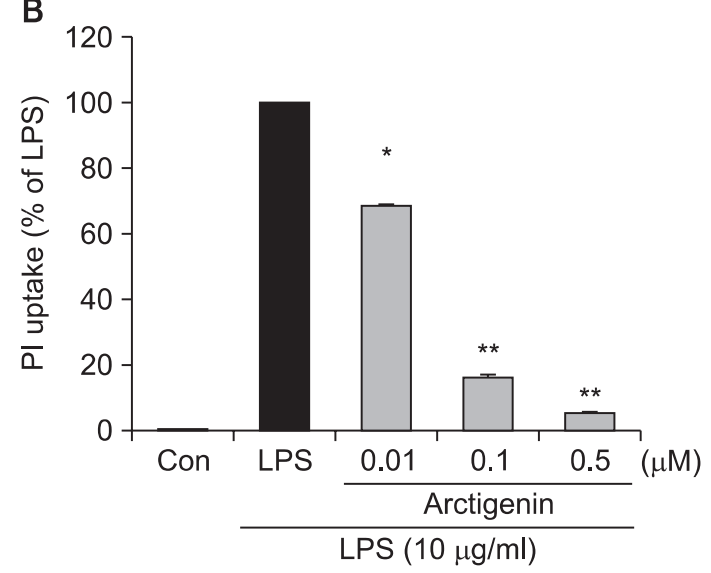

Fig. 3. The effect of arctigenin on neuronal cell death in organotypic hippocampal cultures. Hippocampal slices were pretreated with arctigenin for 30 minutes and stimulated with LPS $(10 \mu \mathrm{g} /$ $\mathrm{ml})$. To investigate the effect of arctigenin on neuronal cell death, we performed PI staining. (A) Neuronal cell death was photographed by $\mathrm{PI}$ fluorescence imaging under a confocal microscope at $514 \mathrm{~nm}$. A representative picture from one of the three different experiments is shown. Bar $=500 \mu \mathrm{m}$. (B) Neuronal cell death was assessed by the PI uptake values. All data are presented as the mean ( \pm SE) of three independent experiments. ${ }^{*} p<0.01$ and ${ }^{* *} p<0.001$ indicate statistically significant differences as compared to treatment with LPS alone.

cause of the systematic difference between a primary cultured tissue and an immortalized cell line. Nevertheless, we suspect that arctigenin, as a neuroprotective agent, may be more potent in an ex vivo system than in an in vitro system. However, investigation in an in vivo system is necessary to confirm these results.

In this study, FF-T also inhibited NO production. Among the FF-T subfractions, FF-E was the only effective fraction. We deduced that a large amount of arctigenin may be contained in $F F-E$, and, as expected, arctigenin was found to be one of the most abundant compounds in FF-E on HPLC analysis. Therefore, arctigenin may play an important role in the suppression of NO production by FF treatment in over-activated microglia. However, the anti-inflammatory effects of other compounds isolated from FF have also been reported. Phylligenin and rengyolone had an anti-inflammatory effect in LPS-stimulated Raw264.7 cells (Kim et al., 2006; Lim et al., 2008). Pinoresinol suppresses inflammatory responses in LPS-activated microglia of rat cerebral cortices (Jung et al., 2010). Therefore, it is possible that the inhibitory activities of FF on NO production may be a result of the synergy between many compounds in
FF.

Because FF inhibits the binding of platelet activating factor to platelets (Iwakami et al., 1992) and can cause extra bleeding, treatment with FF may need to be avoided during and after surgery. Moreover, arctigenin suppresses heat shock response in mammalian cells (Ishihara et al., 2006). Therefore, we suggest that FF and arctigenin should be used carefully.

In conclusion, arctigenin, the active compound in FF, may reduce neuronal cell death by suppressing neuroinflammation in over-activated microglia. Ultimately, arctigenin could be used as a candidate treatment for neuronal damage and various neurodegenerative diseases.

\section{ACKNOWLEDGMENTS}

This research was supported by Korea Institute of Planning and Evaluation for Technology in Food, Agriculture, Forestry and Fisheries (iPET) (No.810006-03-1-SB120). We thank May Kim at Korea International School and Jinhwa Hong at Kyung Hee University for technical assistance.

\section{REFERENCES}

Awale, S., Lu, J., Kalauni, S. K., Kurashima, Y., Tezuka, Y., Kadota, S. and Esumi, H. (2006) Identification of arctigenin as an antitumor agent having the ability to eliminate the tolerance of cancer cells to nutrient starvation. Cancer Res. 66, 1751-1757.

Banati, R. B., Gehrmann, J., Schubert, P. and Kreutzberg, G. W. (1993) Cytotoxicity of microglia. Glia. 7, 111-118.

Boje, K. M. and Arora, P. K. (1992) Microglial-produced nitric oxide and reactive nitrogen oxides mediate neuronal cell death. Brain Res. 587, 250-256.

Choi, I. Y., Moon, P. D., Koo, H. N., Myung, N. Y., Kim, S. J., Lee, J. H., Han, S. H., Moon, G., Seo, S. Y., Sung, H. J., Park, R. K., Jeong, H. J., Um, J. Y., Kim H. M. and Hong, S. H. (2007) Observations of Forsythia koreana methanol extract on mast cell-mediated allergic reactions in experimental models. In Vitro Cell Dev. Biol. Anim. 43, 215-221.

Frotscher, M., Heimrich, B. and Schwegler, H. (1990) Plasticity of identified neurons in slice cultures of hippocampus: a combined Golgi/ electron microscopic and immunocytochemical study. Prog. Brain Res. 83, 323-339.

Frotscher, M., Zafirov, S. and Heimrich, B. (1995) Development of identified neuronal types and of specific synaptic connections in slice cultures of rat hippocampus. Prog. Neurobiol. 45, vii-xxviii.

Gehrmann, J., Matsumoto, Y. and Kreutzberg, G. W. (1995) Microglia: intrinsic immuneffector cell of the brain. Brain Res. Brain Res. Rev. 20, 269-287.

Guo, H., Liu, A. H., Ye, M., Yang, M. and Guo, D. A. (2007) Characterization of phenolic compounds in the fruits of Forsythia suspensa by high-performance liquid chromatography coupled with electrospray ionization tandem mass spectrometry. Rapid Commun. Mass Spectrom 21, 715-729.

Ha, S. K., Lee, P., Park, J. A., Oh, H. R., Lee, S. Y., Park, J. H., Lee, E. H., Ryu, J. H., Lee, K.. R. and Kim, S. Y. (2008) Apigenin inhibits the production of NO and PGE2 in microglia and inhibits neuronal cell death in a middle cerebral artery occlusion-induced focal ischemia mice model. Neurochem. Int. 52, 878-886.

Hanisch, U. K. (2002) Microglia as a source and target of cytokines. Glia. 40, 140-155.

Ishihara, K., Yamagishi, N., Saito, Y., Takasaki, M., Konoshima, T. and Hatayama, T. (2006) Arctigenin from Fructus Arctii is a novel suppressor of heat shock response in mammalian cells. Cell Stress Chaperones 11, 154-161.

Iwakami, S., Wu, J. B., Ebizuka, Y. and Sankawa, U. (1992) Platelet activating factor (PAF) antagonists contained in medicinal plants: 
lignans and sesquiterpenes. Chem. Pharm. Bull. (Tokyo) 40, 11961198.

Jung, H. W., Mahesh, R., Lee, J. G., Lee, S. H., Kim, Y. S. and Park, Y. K. (2010) Pinoresinol from the fruits of Forsythia koreana inhibits inflammatory responses in LPS-activated microglia. Neurosci. Lett. 480, 215-220.

Kaminska, B. (2005) MAPK signalling pathways as molecular targets for anti-inflammatory therapy--from molecular mechanisms to therapeutic benefits. Biochim. Biophys. Acta. 1754, 253-262.

Kang, H. S., Lee, J. Y. and Kim, C. J. (2008) Anti-inflammatory activity of arctigenin from Forsythiae Fructus. J. Ethnopharmacol. 116, 305-312.

Kim, J. H., Kim, D. H., Baek, S. H., Lee, H. J., Kim, M. R., Kwon, H. J. and Lee, C. H. (2006) Rengyolone inhibits inducible nitric oxide synthase expression and nitric oxide production by down-regulation of NF-kappaB and p38 MAP kinase activity in LPS-stimulated RAW 264.7 cells. Biochem. Pharmacol. 71, 1198-1205.

Kim, J. M., Lee, P., Son, D., Kim, H. and Kim, S. Y. (2003a) Falcarindiol inhibits nitric oxide-mediated neuronal death in lipopolysaccharidetreated organotypic hippocampal cultures. Neuroreport 14, 1941 1944.

Kim, M. S., Na, H. J., Han, S. W., Jin, J. S., Song, U. Y., Lee, E. J., Song, B. K., Hong, S. H. and Kim, H. M. (2003b) Forsythia fructus inhibits the mast-cell-mediated allergic inflammatory reactions. Inflammation. 27, 129-135.

Kim, N. Y., Kang, T. H., Song, E. K., Pae, H. O., Chung, H. T. and Kim, Y. C. (2000) Inhibitory effects of butanol fraction of the aque- ous extract of Forsythia koreana on the nitric oxide production by murine macrophage-like RAW 264.7 cells. J. Ethnopharmacol. 73, 323-327.

Lee, J. Y. and Kim, C. J. (2010) Arctigenin, a phenylpropanoid dibenzylbutyrolactone lignan, inhibits type I-IV allergic inflammation and pro-inflammatory enzymes. Arch. Pharm. Res. 33, 947-957.

Lee, P., Hur, J., Lee, J., Kim, J., Jeong, J., Kang, I., Kim, S. Y. and Kim, H. (2006) 15,16-dihydrotanshinone I suppresses the activation of BV-2 cell, a murine microglia cell line, by lipopolysaccharide. Neurochem. Int. 48, 60-66.

Lim, H., Lee, J. G., Lee, S. H., Kim, Y. S. and Kim, H. P. (2008) Antiinflammatory activity of phylligenin, a lignan from the fruits of Forsythia koreana, and its cellular mechanism of action. J. Ethnopharmacol. 118, 113-117.

Minghetti, L. and Levi, G. (1998) Microglia as effector cells in brain damage and repair: focus on prostanoids and nitric oxide. Prog. Neurobiol. 54, 99-125.

Miyake, K. (2004) Innate recognition of lipopolysaccharide by Toll-like receptor 4-MD-2. Trends Microbiol. 12, 186-192.

Perry, V. H. and Gordon, S. (1988) Macrophages and microglia in the nervous system. Trends Neurosci. 11, 273-277.

Piao, X. L., Jang, M. H., Cui, J. and Piao, X. (2008) Lignans from the fruits of Forsythia suspensa. Bioorg. Med. Chem. Lett. 18, 19801984.

Yang, Z., Liu, N., Huang, B., Wang, Y., Hu, Y. and Zhu, Y. (2005) Effect of anti-influenza virus of Arctigenin in vivo. Zhong Yao Cai. 28, 1012-1014. 